\title{
Plantas medicinales en una plantación de cacao en Guápiles, Costa Rica
}

\author{
Agustín Contreras Arias ${ }^{1}$ y Christoph Campregher ${ }^{2}$ \\ 'Maestría en Manejo de Recursos Naturales, Universidad Estatal a Distancia, Sabanilla, Montes de Oca, San José, Costa Rica; etnoarias@gmail.com \\ ${ }^{2}$ Maestría en Manejo de Recursos Naturales, Universidad Estatal a Distancia, Sabanilla, Montes de Oca, San José, Costa Rica y Universidad de Viena, \\ Austria; campregher@gmx.at
}

Recibido 5-IV-2010 Corregido 3-VI-2010 Aceptado 11-VI-2010

\begin{abstract}
Medicinal plants in a cocoa plantation in Guápiles, Costa Rica. Costa Rica has a rich medicinal flora of several hundred species. These plants grow in several types of areas, some of which are cultivated. We document the diversity of species in and around a plantation of organic cocoa in Guácimo, Limón, Costa Rica. The plantation produces organic cacao which is exported to Europe. The local population -which lives and works on the plantation- collects a total of 61 medicinal plant species in their home gardens, cocoa plantations, border areas between plantations and roads, and riparian forests inside and near the plantation. None of these plants were found in all four areas. Gardens had the highest diversity. This confirms the importance of gardens and poli-cultivated fields for the conservation of agro-biodiversity and biocultural diversity. Furthermore, we documented 10 species with other important uses, of which one is in danger of extinction. The local population does not have specific knowledge on the preparation and effects of many medicinal plants in their areas: education is needed in this area.
\end{abstract}

\section{KEY WORDS}

Medicinal plants, plant diversity, ethnobotany, biocultural diversity, cacao plantation, Guápiles.

\section{RESUMEN}

Costa Rica tiene una flora rica en plantas con usos medicinales. El presente trabajo documenta la variedad de plantas con uso medicinal y otros usos importantes dentro y alrededor de una plantación de cacao orgánico en Pueblo Nuevo de Guácimo, Costa Rica. En un sondeo rápido encontramos un total de 61 especies con valores medicinales en cuatro categorías de áreas utilizadas por la población local, jardines o huertas caseras, las orillas entre caminos y plantaciones y sus cercas vivas, las plantaciones de cacao orgánico y bosques ribereños. Además, se identificaron 10 especies con otros usos importantes, una de las cuales se encuentra en peligro de extinción. Sin embargo, a pesar de la gran variedad de plantas, es importante hacer notar que son subutilizadas por la población local, la cual no tiene conocimiento específico sobre medicina natural con la excepción de algunas plantas de uso popular. Por esta razón sugerimos que autoridades y la sociedad civil promuevan programas de educación ambiental para promover el uso de la medicina tradicional en las áreas rurales.

\section{PALABRAS CLAVE}

Plantas medicinales, agrobiodiversidad, etnobotánica, diversidad biocultural, plantación de cacao, Guápiles.
En Costa Rica, el uso de la diversidad de plantas autóctonas e introducidas por campesinos y campesinas se contrasta con la agricultura en monocultivos, promovida por empresas agroindustriales. Un aspecto importante de la agricultura tradicional campesina es la conservación de especies de plantas medicinales (Ammour et al. 1996, Fernández 2007). Costa Rica pose una flora rica de más de 400 especies medicinales en 106 géneros (Ocampo 1997). Sin embargo, actualmente se está perdiendo mucho del conocimiento tradicional y popular sobre los usos medicinales de la flora (Kappelle et al. 2000). Ante esta situación preocupante, es importante documentar la diversidad de plantas medicinales que existe en Costa Rica y su utilización por parte de la población campesina e indígena. Basándonos en este conocimiento debemos promover el cultivo y aprovechamiento de estas plantas, especialmente de las especies autóctonas.

Plantas medicinales crecen en áreas silvestres y son producidas por los costarricenses en tres tipos de cultivos: huertos caseros, huertos de mercado y fincas comerciales (Ocampo 1997). En el estudio de caso presente documentamos las especies florísticas con valor medicinal 
en diferentes sitios en una plantación de cacao y sus alrededores. Metodológicamente, nos enmarcamos en los estudios interdisciplinarios sobre la conservación de la diversidad biocultural (Carlson \& Maffi 2004, Maffi 2005) en Centroamérica utilizando métodos botánicos y etnobotánicos (Martin 1995, Vogl et al. 2004). El conocimiento de la distribución y diversidad de la flora local y su uso es un requisito importante para la conservación del conocimiento tradicional y el manejo sostenible de la agro-biodiversidad (Zaldivar et al. 2002). Su investigación genera posibilidades de crear nuevas fuentes de ingresos económicos. Además, las plantas medicinales enriquecen la subsistencia de las familias en zonas rurales (Fernandes \& Nair 1986, Padoch \& de Jong 1991, Watson \& Eyzaguirre 2002). Por está razón, una parte importante del estudio presente consistía en recopilar información sobre los diversos usos medicinales que tenga la flora presente en el área de estudio por medio de la revisión de literatura disponible y la consulta con expertos, para luego resumir esta información para las personas en el área de estudio. ¿Cuáles plantas se encuentran en diferentes áreas dentro y alrededor de la plantación de cacao orgánico? ¿Y cuales usos medicinales tienen estas plantas? ¿Como la población local las sabe aprovechar?

\section{METODOLOGIA}

La recolección de datos se realizó en un sondeo rápido (Martin 1995) durante un periodo de tres días en una plantación de cacao orgánico (Theobroma cacao, familia Sterculiaceae) localizada en Pueblo Nuevo de Guápiles, Provincia de Limón. La finca se Ilama FINMAC S.A. y tiene una extensión de 120 hectáreas. Se ubica en una zona del trópico húmedo premontano en las coordenadas $10^{\circ} 19^{\prime} 19^{\prime \prime} \mathrm{N}, 83^{\circ} 35^{\prime} 27^{\prime \prime} \mathrm{W} ; 10^{\circ} 19^{\prime} 22^{\prime \prime} \mathrm{N}, 83^{\circ} 35^{\prime} 36^{\prime \prime} \mathrm{W}$; y $10^{\circ} 19^{\prime} 19^{\prime \prime} \mathrm{N}, 83^{\circ} 35^{\prime} 36^{\prime \prime} \mathrm{W}$. Alrededor de esta finca de cacao se encuentran plantaciones monocultivos de piña (este), banano (oeste) y potreros con árboles aislados (noreste y sureste). También, se encuentran pequeños parches de bosque secundario y bosque ribereño a lo largo de las orillas de los ríos y canales artificiales.

Para la recolección de datos, se empezó a recorrer el sitio con el fin de conocer las condiciones y dimensiones espaciales del terreno. Luego, se consultó con la población que vive y labora en la finca sobre sitios donde se puede encontrar especies de valor medicinal según su criterio. De acuerdo a las respuestas obtenidas y a las condiciones del lugar, se decidió muestrear las diferentes áreas, principalmente jardines, plantaciones de cultivos y áreas boscosas.
En estos sitios se muestreó todas las plantas florísticas con valor medicinal según el saber de los investigadores o el conocimiento de la población consultada. En el caso de los jardines, se muestreó todas las jardines de la población que vive dentro de la finca. Las otras dos categorías de sitios fueron recorridos con un informante y con base en la información obtenida de la población local para encontrar las especies mencionadas. La mayoría de especies fueron identificadas en el sitio. Se llevaron muestras de especimenes únicamente de las plantas que no se pudieron identificar in situ para su identificación posterior, para la cual se consultó libros de referencia y herbarios virtuales. Para determinar el conocimiento sobre plantas medicinales por parte de la población de la finca, se realizaron entrevistas etnográficas (Barrantes 2008), no estructuradas con preguntas abiertas a diferentes personas.

\section{RESULTADOS}

\section{Distribución y presencia de plantas medicinales}

Hay cinco familias que viven en la finca FINMAC. Ellos habitan casas cerca a la entrada principal. De acuerdo a las personas entrevistadas, no existe ninguna persona que tenga conocimiento específico sobre el uso de plantas medicinales. Por lo contrario, ellos afirman que utilizan algunos plantas de uso popular como el jengibre (Zingiber officinale), la noni (Morinda citrifolia), verdolaga (Portulaca oleracea), uña de gato (Uncaria tomentosa), entre otros, pero que no conocen mucho sobre los efectos medicinales de otras plantas que existen en la zona.

Las plantas que utilizan, las encuentran en áreas que se puede dividir en cuatro categorías; (1) sus jardines o huertas caseras, (2) en las orillas de caminos entre plantaciones de cacao y porteros, (3) las plantaciones de cacao orgánico (4) las orillas de ríos y canales (bosque ribereños) (Cuadro 1). En un muestreo rápido de estas áreas encontramos un total de 61 plantas con valores medicinales científicamente reconocidos (Brown 1995, León 2000, Fernández 2007, entre otros).

La mayoría de las familias que viven dentro de la plantación llegó de otras zonas del país durante los últimos veinte años. El motivo principal de esta migración era la busca de empleo. Ellos habitan casas de cemento y utilizan el espacio a su alrededor para cultivar plantas ornamentales, comestibles y medicinales, para la cría de animales y para fines de recreación. Cada familia dispone de un terreno de aproximadamente 15 por 10 metros, frente a su casa, que utiliza para sembrar diferentes cultivos para el autoconsumo, principalmente para uso alimenticio. Estos jardines son de diferentes edades y varían en su 


\section{CUADRO 1}

Distribución de plantas medicinales en diferentes áreas.

\begin{tabular}{|c|c|c|c|c|}
\hline Planta & Jardín & Orilla de camino & Cacao & $\begin{array}{l}\text { Bosque } \\
\text { Ribereño }\end{array}$ \\
\hline Acalypha spp. & & $x$ & & \\
\hline Annona muricata & $\mathrm{X}$ & & & \\
\hline Azadirachta indica & & $x$ & & \\
\hline Bixa orellana $\mathrm{L}$. & $\mathrm{x}$ & & & \\
\hline Capsicum spp. & $x$ & & & \\
\hline Carica papaya & $X$ & & & \\
\hline Castilla elastica & & & & $x$ \\
\hline Cecropia peltata & & & & $x$ \\
\hline Caesalpinia pulcherrima & & $x$ & & \\
\hline Cedrela odorata & & $x$ & & $x$ \\
\hline Cestrum racemosum & & $x$ & & \\
\hline Costus spp. & $x$ & & & $x$ \\
\hline Crecentia cujete & $x$ & & & \\
\hline Cucurbita pepo & $\mathrm{X}$ & & & \\
\hline Cyathula spp. & & & $x$ & \\
\hline Cymbopogon citratus & $x$ & & & \\
\hline Desmodium spp. & & $x$ & & \\
\hline Dicliptera unguiculata & $x$ & & & \\
\hline Dioscorea spp. & $x$ & & & \\
\hline Erythrina spp. & $x$ & $x$ & $x$ & \\
\hline Eugenia stipitata & $x$ & & & \\
\hline Gliricidia sepium & $x$ & & & \\
\hline Goethalsia meiantha & & & & $x$ \\
\hline Hamelia patens & $\mathrm{X}$ & & & $x$ \\
\hline Hampea appendiculata & & & & $x$ \\
\hline Hibiscus rosa-sinensis & $\mathrm{x}$ & & & \\
\hline Lantana camara & & $x$ & & \\
\hline Leucaena leucocephala & & & $x$ & \\
\hline Lippia alba & $x$ & & & \\
\hline Lippia graveolens & $x$ & & & \\
\hline Ludwigia spp. & $x$ & $x$ & & \\
\hline Mangifera indica & $x$ & & & \\
\hline Manihot esculenta & $x$ & & & \\
\hline Mimosa pudica & & $x$ & & $x$ \\
\hline Momordica charantia & $x$ & & $x$ & \\
\hline Morinda citrifolia & $x$ & & & \\
\hline Morus alba & $x$ & & & \\
\hline Opuntia cochenillifera & & $\mathrm{X}$ & & \\
\hline
\end{tabular}


CUADRO 1 (Continuación...)

Distribución de plantas medicinales en diferentes áreas.

\begin{tabular}{|c|c|c|c|c|}
\hline Planta & Jardín & Orilla de camino & Cacao & $\begin{array}{l}\text { Bosque } \\
\text { Ribereño }\end{array}$ \\
\hline Passiflora spp. & & $x$ & & \\
\hline Persea americana & $x$ & & & \\
\hline Piper peltatum & $x$ & & & $x$ \\
\hline Phyllantus urinaria-niruri & $x$ & $x$ & & $x$ \\
\hline Piper auritum & & & & $x$ \\
\hline Portulaca oleracea & & $x$ & & \\
\hline Posoqueria latifolia & & & & $x$ \\
\hline Protium spp. & & & & $x$ \\
\hline Pseudelephantophus spicatus & $x$ & & & \\
\hline Psidium guajava & $x$ & $x$ & & \\
\hline Quassia amara & $x$ & & & \\
\hline Rivina humilis & & $x$ & $\mathrm{x}$ & \\
\hline Sida acuta & & $x$ & & \\
\hline Solanum americanum & & & & $x$ \\
\hline Solanum mammosum & & & & $x$ \\
\hline Spondias mombin & & & & $x$ \\
\hline Spondias purpurea $\mathrm{L}$ & $x$ & & & \\
\hline Tecoma stans & & $x$ & & \\
\hline Theobroma cacao & & & $x$ & \\
\hline Thunbergia fragans & & & $x$ & \\
\hline Urera baccifera & & $x$ & $x$ & $x$ \\
\hline Zingiber officinale & $x$ & & & \\
\hline Zygia longifolia & $x$ & & & $x$ \\
\hline Total & 32 & 19 & 8 & 18 \\
\hline
\end{tabular}

composición y diversidad de cultivos. Se encontró un total de 32 especies con valor medicinal en los cinco jardines que se muestreó. Es importante anotar que sus dueños (o las personas que encontramos en las casas ya que en ningún caso se encontraron todos los miembros de la familia a la hora de las entrevistas) en muchos casos desconocían de estos aspectos medicinales. En otros casos sabían que tienen valor medicinal pero no sabían como procesar, preparar y utilizar las plantas.

La gran mayoría del área dentro de la plantación orgánica está ocupada por monocultivos de cacao (Theobroma cacao) con algunas especies de árboles introducidas que le brindan sombra (Eucalyptus spp.). El cultivo orgánico implica en este caso que se este eliminando constantemente las hierbas que crecen en el estrato bajo de las plantaciones. El efecto de control de hierbas, que ayuda a la disminución de plagas en las plantas, por ende, causa un efecto de baja diversidad de especies bajo la sombra del cacao. Por esta razón, encontramos solamente ocho especies con valor medicinal en el recorrido de esta área.

En las orillas del camino dentro de la plantación se registraron 19 especies de plantas medicinales, es decir una mayor diversidad comparado con la plantación de cacao. Algunas de estas especies están utilizadas en cercas vivas. 
En el transecto recorrido entre jardines y plantaciones de cacao, las cercas eran dominadas por madero negro (Gliricidia sepium) y poró (Erythrina spp.). En el área recorrido de cacao y bosque ribereño, la especie que dominaba en las cercas vivas era el ním (Azadirachta indica). Muchas de las otras especies que se encontró crecen debajo de las cercas.

Finalmente, recorrimos bosques ribereños en las orillas de un canal dentro de la plantación y un río en su cercanía. Al estar menos alterada en control de hierbas, en esta área se podía documentar la existencia de plantas que se eliminan dentro del área de producción de la finca. En estos bosques encontramos 18 especies de plantas medicinales, alimenticias o con otro uso potencial de su fibra. Diez de estas plantas no se encuentra en ninguno de las otras áreas muestreadas.

\section{Otras especies no medicinales}

Queremos mencionar otras especies ecológicamente importantes que se pudieron encontrar en el bosque ribereño. Entre las que podemos rescatar es el árbol de titor o campano (Sacoglotttis trichogyna) de la familia humiricaceae. La importancia de esta planta se debe a que suministra alimento a la lapa verde (Ara ambigua) de acuerdo a observaciones de la población local.

Otro árbol de importancia es el molenillo (Quararibea asterolepis) de la familia malvaceae. Los frutos de este árbol alimentan a una gran variedad de fauna, como al mono cariblanco (Cebus capucinus), aves como trogones, aracaris, y ciertas especies de murciélagos. Unos de los primates observados que utilizaba este bosque ribereño fue el mono congo (Alouatta palliata).

Otro árbol importante de anotar por estar en peligro de extinción, es el (Lecythis ampla. Miers, Lecithydaceae) conocido como olla de mono. Este árbol se encontró aislado del resto del bosque ribereño en un potrero al este de la finca. Las semillas de esta especie alimentan a roedores como las guatusas (Dasyprocta punctata) y tepezcuintles (Agouti paca). Otras especies de árboles emergentes observadas son el sangrillo (Pterocarpus officinalis) y la fruta dorada (Virola sebifera).

\section{DISCUSIÓN}

Ninguna especie con valor medicinal está presente en todas las áreas muestreadas, y por lo contrario, la mayoría de especies se encuentra solamente en una de las áreas, lo cual afirma la importancia de contar con paisajes y cultivos variados para conservar la diversidad botánica y agro-biológica. Sin embargo, por la falta de conocimiento esta diversidad de plantas esta subutilizada por la población local.

El total de especies encontradas en el área de jardín es de 32 especies, las cuales están distribuidos de la siguiente manera: Anacardiaceae ( 2 especies), Annonaceae (1), Acanthaceae (1), Asteraceae (1), Bignoniaceae (1), Bixaceae (1), Caricaeae (1), Costaceae (1), Cucurbitaceae (2), Dioscoreaceae (1), Euphorbiaceae (2), Lauraceae (1), Malvaceae (1), Mimosoideae (1), Moraceae (1), Myrtaceae (2), Onagraceae (1), Papilionoideae (2), Piperaceae (1), Poaceae (1), Rubiaceae (2), Simaroubaceae (1), Solanaceae (1), Verbenaceae (2), Zingiberaceae (1).

Hay que recalcar que muchas de las plantas en los jardines están por el valor que se les atribuye ya sea como medicina o alimento. Un ejemplo es la Lippia graveolens conocido como orégano mexicano o de palo, el cual no es nativo y usualmente prefiere zonas que presenten una época seca bien marcada.

También existen especies que crecen en áreas como los huertos pero son consideradas malas hierbas. Una de estas, es el Phyllanthus urinaria o Phyllanthus niruri que tiene gran uso en América del Sur como planta para tratar piedras de biliares y del riñón. Este uso no es conocido por las familias que viven en la finca.

Con respecto a las plantas presentes en las orillas de los caminos entre jardines y cacao, así como cacao y bosque ribereño, la distribución de plantas por familia es la siguiente: Bignoniaceae (1 especie), Cactaceae (1), Caesalpinioideae (1), Euphorbiaceae (2), Malvaceae (1), Meliaceae (2), Mimosoideae (1), Myrtaceae (1), Onagraceae (1), Papilionoideae (2), Passifloraceae (1), Phytolaccaceae (1), Portulacaceae (1), Solanaceae (1), Urticaceae (1), Verbenaceae (1),

Entre estas especies hay una en particular que se encuentra por el valor que se le dio como uso de insecticida natural. El neem o nim (Azaderachta indica) de la familia de las Meliaceae, es una planta introducida desde la India. Sin embargo, las condiciones climáticas de la zona no son las adecuadas para su desarrollo ya que prefiere un clima más seco. Esta planta no es valorada por su valor medicinal por parte de la población local, a pesar de que tiene una gran gama de beneficios para la salud para los que saben aprovechar sus propiedades. Otra especie es el Cestrum recemosum de la familia Solanaceae que tiene valor alimenticio.

La distribución de las especies medicinales potenciales según familia en el área de la plantación de caco es la siguiente: Acanthaceae (1 especie), Amaranthaceae (1), Cucurbitaceae (1), Mimosoideae (1), Papilionoideae (1), Phytolaccaceae (1), Sterculiaceae (1), Urticaceae (1). 
Hay que mencionar que de las 8 especies encontradas solo 4 especies se pueden considerar de uso medicinal en el sentido estricto (Theobroma cacao, Momordica charantia, Urera baccifera, Erythrina spp.). Los demás tienen otros usos importantes.

En esta área se resalta el sorosí o pepinillo amargo (Momordica charantia) por su valor medicinal. Esta planta tiene alto potencial para el tratamiento de diabetes tipo 2 .

Por último, en el área del bosque ribereño encontramos especies de plantas silvestres que suministren medicina, alimento, resina, goma y fibra, entre otros. Algunos de estas no se encontraron en las áreas analizadas anteriormente, lo cual afirma la importancia de contar con áreas naturales para conservar la diversidad biológica y el conocimiento medicinal de plantas. Se observó la presencia de especies de las familias siguientes: Anacardiacea (1 especie), Burseraceae (2), Cecropiaceae (1), Costaceae (1), Euphorbiaceae (1), Malvaceae (1), Meliaceae (1), Mimosoideae (2), Moraceae (1), Piperaceae (1), Rubiaceae (2), Solanaceae (2), Tiliaceae (1), Urticaceae (1).

Entre las plantas que se destacan como medicinal está el pichichillo (Solanum mamosum) y la yerba mora (Solanum americanum). El primero se ha utilizado para tratamiento de sinusitis, que frecuentemente afecta a la población en zonas con mucha humedad. Además, tiene otro uso potencial como controlador natural de roedores y cucarachas utilizando el fruto como sebo.

Entre las especies determinadas, encontramos al burio (Hampea appendiculata) familia Malvaceae. De esta planta se utiliza la corteza para la fabricación de cuerdas y la confección de artesanías como bolsos.

Como planta resinosa encontramos al canfín (Protium spp.), familia Burseraceae. Este género se caracteriza por producir una resina aceitosa de agradable olor que puede ser utilizada para encender fuegos. En la medicina natural, la resina es utilizada para sanar granos o llagas de la piel.

Es difícil comparar los resultados del estudio presente con otros casos por las metodologías diferentes que se utilizan en cada uno. Aplicando una encuesta etnobotánica, Kapelle et al. (2000) encontraron 45 plantas con usos medicinales conocidos por las personas en San Gerardo de la Dota, en jardines y plantaciones en la zona de los Santos. Estos investigadores razonan que la diversidad de plantas medicinales que encontraron es baja por la falta de conocimiento por parte de la población. Por el otro lado, en talleres participativos con los Bribri y Cabécares se documentó el uso de 283 especies diferentes en sus fincas, las cuales usan como medicina (104), comestibles (103), para construcción (63), artesanía (55), leña (42), comercio (28), tintes (8) y ornamentos (6) (Trujilla-Córdova et al. 2003). Una vez mas se afirma por medio de estos estudios la fuerte relación entre el conocimiento tradicional por parte de poblaciones locales y la diversidad de especies biológicas que se encuentran en sus áreas cultivadas (Carlson \& Maffi 2004, Maffi 2005).

Encontramos 61 especies diferentes que se pueden utilizar con fines medicinales en la plantación de cacao orgánico FINMAC y sus alrededores. Estas especies se encuentran en cuatro categorías de áreas: jardines, plantaciones de cacao, orillas de camino y cercas entre plantaciones, así como los bosques ribereños. El área con mayor diversidad en especies con potencial medicinal son los jardines de la población que labora en la finca. Se afirma la importancia de los jardines y huertos familiares para la conservación de la diversidad botánica y agrológica (Zaldivar et al. 2002, Vogl et al. 2004, Maffi 2005).

Es importante recalcar ante los pobladores y los que interactúan con la finca FINMAC los posibles usos de las plantas que se encuentran dentro de esta finca y a sus alrededores. Ofrecerles información y capacitación en cómo reconocer y utilizar las valiosas propiedades que ofrece la flora del bosque lluvioso tropical, es clave para crear una conciencia que valora el medio ambiente.

Además de las plantas medicinales se identificaron otras plantas de uso importante para la población humana y la fauna de la región. Algunos son considerados especies en peligro de extinción. Otros se destacan por los usos múltiples que se les puede dar. Hay plantas medicinales y comestibles que solamente se encuentran en áreas de vegetación natural como los bosques ribereños. De acuerdo a la experiencia de uno de los autores con los indígenas Ngöbe, estas poblaciones todavía conservan un conocimiento tradicional sobre el uso de plantas silvestres (Campregher, entrevistas y observación). Sugerimos que se debe investigar sobre la distribución del conocimiento entre diferentes sectores de la población costarricense. Estos estudios podría ser complementadas mediante programas para promover y redistribuir este conocimiento desde las poblaciones que más lo han conservado hacía otros sectores de la sociedad.

Para este fin, es importante elaborar estudios etnobotánticos y etnoecológicos más sofisticados que el presente. Reconocemos las limitaciones del estudio presente, es decir el período corto del sondeo rápido. Tampoco se estandardizaron las áreas muestreadas, lo cual impide un análisis estadístico sistemático y más detallado. Para estudios a futuro, sugerimos plantear y probar hipótesis diferentes sobre la distribución de plantas útiles en el espacio y sobre la distribución y conservación del conocimiento asociado por parte de la población costarricense. Esto requiere la cooperación interdisciplinaria entre científicos naturales (como los botánicos y biólogos) y sociales (como antropólogos y sociólogos). 


\section{AGRADECIMIENTOS}

Agradecemos el apoyo que recibimos por parte del Programa de Maestría en Manejo de Recursos Naturales y sobre todo de Oscar Ramírez. También agradecemos a las personas que viven en FINMAC por su hospitalidad y su disposición de contestar nuestras preguntas. La estadía de Christoph Campregher en Costa Rica fue financiada parcialmente por una beca KWA de la Universidad de Viena, Austria.

\section{REFERENCIAS}

Ammour, T., R. Ocampo \& G. Robles. 1996. Caracterización de los sectores asociados la producción, comercialización y transformación de plantas medicinales en Costa Rica. Memorias del X Congreso Nacional Agronómico.

Barrantes, R. 2008. Investigación: Un Camino al Conocimiento, un enfoque cualitativo y cuantitativo. EUNED. San José, Costa Rica.

Brown, D. 1995. Encyclopedia of herbs and their uses. Dorling Kindersley. Londres, Gran Bretaña.

Carlson, J. \& L. Maffi. 2004. Ethnobotany and Conservation of Biocultural Diversity. Advances in Economic Botany. The New York Botanical Garden. Nueva York, E.E.U.U.

Fernandes, E. \& P. Nair. 1986. An evaluation of the structure and function of tropical homegardens. Agricultural Systems 21: $279-310$

Fernández, P. 2007. Plantas medicinales: saberes ancestrales de la medicina autóctona. Fundación Museos del Banco
Central de Costa Rica. San José, Costa Rica.

Kappelle, M., G. Avertin, M. Juárez \& N. Zamora. 2000. Useful Plants Within a Campesino Community in a Costa Rican Montane Cloud Forest. Mountain Research and Development 20: 162-171.

León, J. 2000. Botánica de los cultivos tropicales. Instituto Interamericano de Cooperación para la Agricultura. 3a edición. Editorial Agroamérica. San José, Costa Rica.

Maffi, L. 2005. Linguistic, Cultural, and Biological Diversity. Annual Review of Anthropology 34: 599-618

Martin, G. 1995. Ethnobotany: a methods manual. People and plants conservation manuals. Chapman \& Hall. Londres, Gran Britana.

Ocampo, R. 1997. Estado de Avance en la Agroindustria de Plantas Medicinales en Costa Rica. Agronomía Costarricense 21: 103-109.

Padoch, C. \& W. De Jong. 1991. The house gardens of Santa Rosa: Diversity and variability in an Amazonian agricultural system. Economic Botany 45: 166-75.

Trujillo-Córdova, L., E. Somarriba-Chávez \& C. Harvey. 2003. Plantas útiles en las fincas cacaoteras de indígenas Bribrí y Cabécar de Talamanca, Costa Rica. Agroforestería en las Américas. 10: 36-41.

Vogl, C., B. Vogl-Lukasser \& R. Puri. 2004. Tools and methods for the ethnobotanical study of gardens. Field Methods 16: 285-306.

Zaldivar, M., O. Rocha, E. Castro \& R. Barrantes. 2002. Species diversity of edible plants grown in homegardens of Chibchan Amerindians from Costa Rica. Human Ecology 30: 301-316. 